\title{
Theoretical Study on the ATP Hydrolysis Mechanism of HisP Protein, the ATP-Binding Subunit of ABC Transporter
}

\author{
Qiang Pei ${ }^{1, * 1}$, Carlos A. Del Carpio ${ }^{1}$, Hideyuki Tsuboi ${ }^{1}$, Michihisa Koyama ${ }^{1}$, \\ Akira Endou ${ }^{1}$, Momoji Kubo ${ }^{1,2}$, Ewa Broclawik ${ }^{3}$, Kazumi Nishijima ${ }^{4,5}$, \\ Tetsuya Terasaki ${ }^{4,6}$ and Akira Miyamoto ${ }^{1,4, * 2}$ \\ ${ }^{1}$ Department of Applied Chemistry, Graduate School of Engineering, Tohoku University, Sendai 980-8579, Japan \\ ${ }^{2}$ PRESTO, Japan Science and Technology Agency, Kawaguchi 332-0012, Japan \\ ${ }^{3}$ Institute of Catalysis and Surface Chemistry, Polish Academy of Sciences, Niezapominajek, 30-239 Cracow, Poland \\ ${ }^{4}$ New Industry Creation Hatchery Center, Tohoku University, Sendai 980-8579, Japan \\ ${ }^{5}$ Clinical Research and Development Division, Mochida Pharmaceutical Co. Ltd., Tokyo 160-0004, Japan \\ ${ }^{6}$ Department of Membrane transport and drug targeting, Graduate School of Pharmaceutical Sciences, \\ Tohoku University, Sendai 980-8578, Japan
}

ATP binding subunit is known as a subunit of ABC transporter, providing energy through the binding of ATP with the subunit and the subsequent hydrolysis reaction of the bound ATP. In this study, density functional theory (DFT) method was used to study the ATP hydrolysis reaction in HisP protein, an ATP binding subunit of Histidine permease HisQMP 2 , by considering the ATP binding site, especially the $\gamma$ phosphate group, surrounding residues and water molecules. Based on DFT calculations, we proposed that ATP hydrolysis is initiated by the formation of $\mathrm{Mg}^{2+}$ mediated coordinate complex followed by the nucleophilic attack of a single water molecule (Water437) on the $\gamma$-phosphate; the hydrolysis product ADP acts as a leaving group. The transition state structure was determined by an approximate saddle-point search. [doi:10.2320/matertrans.48.735]

(Received January 4, 2007; Accepted February 7, 2007; Published March 25, 2007)

Keywords: adenosine-triphosphate hydrolysis, HisP protein, adenosine-tripohosphate binding cassette transporter, chemical reaction mechanism, transition state, density function theory calculation

\section{Introduction}

ATP binding cassette $(\mathrm{ABC})$ transporters constitute one of the largest superfamily of proteins in living organisms and mediate the traffic of molecules across membrane lipid bilayer, which is central to cell physiology. ${ }^{1)}$ ABC transporters occupy almost $5 \%$ of the genome in humans. ${ }^{2)}$ Mutations in $\mathrm{ABC}$ transporter genes are the cause of many diseases. ${ }^{3)}$ Numerous investigations have been carried out on $\mathrm{ABC}$ transporters to understand the behavior of this important protein family. ${ }^{4-12)}$ However, the mechanism of conformational changes induced by the binding of ATP in wild type and mutant proteins, functions of important conserved residues and mechanism of ATP hydrolysis in ABC transporters still remain unclear.

The membrane bound complex of a typical ABC transporter has four domains, two trans-membrane domains (TMDs) and two nucleotide-binding domains (NBDs). ${ }^{2)}$ NBDs couple conformational changes induced by ATP binding, ATP hydrolysis and ADP release to the transport process, which acts as the switch of open-state or closed-state of $\mathrm{ABC}$ transporters as proposed by Higgins et al. ${ }^{2)}$

One of the first NBD structures, which have been determined and analyzed, is ATP binding subunit of histidine permease $\left(\mathrm{His}_{2} \mathrm{MP}_{2}\right)$, i.e., HisP protein. ${ }^{13)}$ Moreover, it was co-crystallized with ATP, therefore it has become the prototype for theoretical investigation on ATP binding and hydrolysis induced conformational changes.

\footnotetext{
${ }^{* 1}$ Graduate Student, Tohoku University

*2Corresponding author, E-mail: miyamoto@aki.che.tohoku.ac.jp
}

As shown in Fig. 1(a), the overall shape of the HisP protein is that of a letter "L" with two thick arms, and these are usually designated as arms I and II. ${ }^{13)}$ ATP molecule binds at the end of arm I. The conserved sequence called "phosphatebinding loop" or "p-loop" was found in the ATP-binding site, which corresponds to the Walker's A/B motifs, and includes residues Gly39 to Lys45, which link $\beta 3$ to $\alpha 1{ }^{13)}$ The segment corresponding to residues Gly42 to Ser46 interacts with $\beta$-phosphate of the ATP molecule through hydrogen bonding (Fig. 1(b)). Water407 (Wat407), which locates at the position that is usually occupied by the divalent cation required for ATP hydrolysis, interacts with Asp178 through hydrogen bonding. Another water molecule, Water437 (Wat437), which is considered as an attacking nucleophile in the reaction of ATP hydrolysis, interacts with Gln100 and Glu179 through hydrogen bonding. ${ }^{13)}$ Several computational studies addressing the conformational changes induced by ATP binding have been carried out for this protein using molecular dynamics (MD) method. They reported that the conformational changes within HisP, i.e., a rotation of three $\alpha$ helices $(\alpha 1, \alpha 2$ and $\alpha 3)$, a movement of signature sequence toward the bound ATP and a considerable flexibility of ploop, are depended on the presence of ATP and are sensitive to the presence/absence of $\mathrm{Mg}^{2+}$ ions bound to ATP. ${ }^{11,12)}$ However, the mechanism of ATP hydrolysis and the several conformational changes induced by this bio-chemical process, including the effects of the protein environment, still remain unclear.

In the present study, we have conducted a series of computational studies oriented to examine the ATP hydrolysis reaction within the ATP binding pocket of HisP protein. 


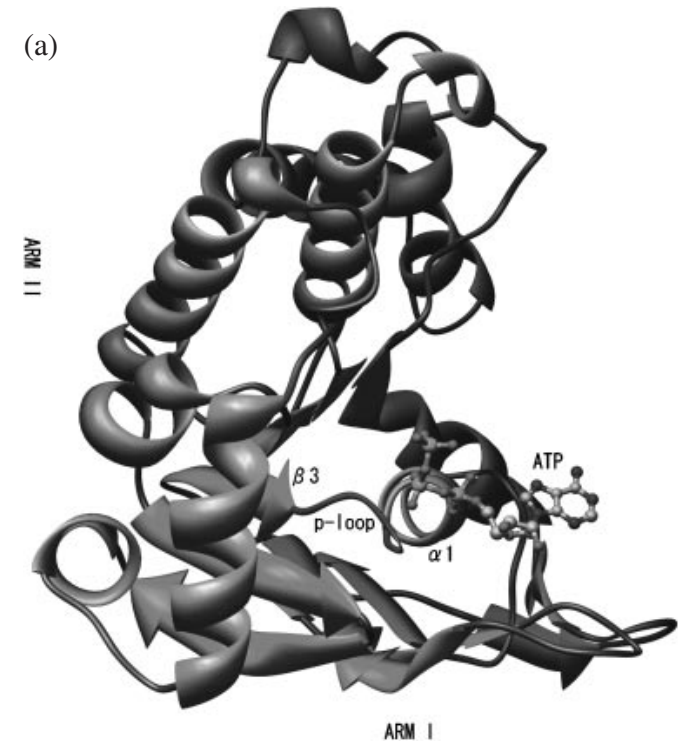

(b)

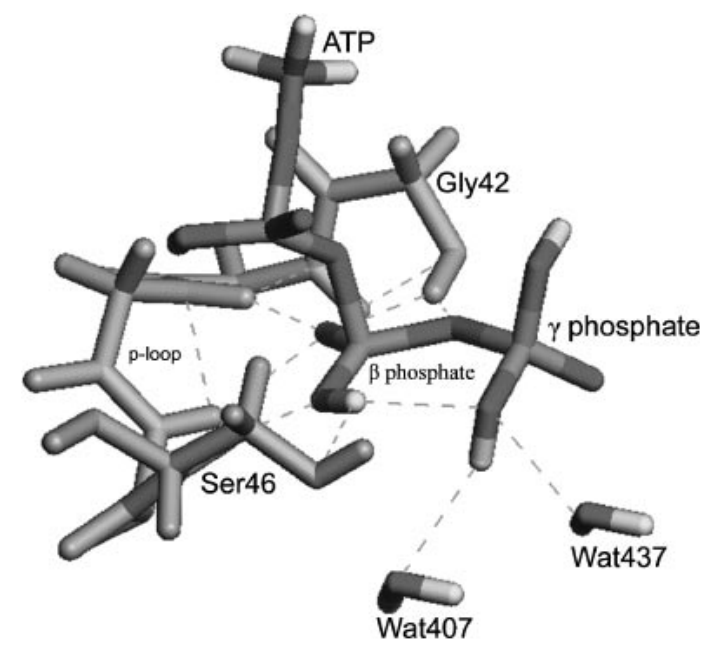

Fig. 1 (a) Ribbon representative of HisP protein with two thick arms I and II. P-loop corresponds to Walker's A/B motifs, which is involved in ATP binding site, connects helix $\alpha 1$ with strand $\beta 3$; ATP molecule is shown in ball and stick representative. (b) Binding site of HisP protein. The P-loop composes residues from Gly42 to Ser46; dot lines represent hydrogen bonding among the binding site.

We have sought to determine the reaction path and thermodynamics of the process.

\section{Methods}

The energy-minimized structure of ATP co-crystallized HisP protein structure, which is extracted from PDB (1B0U), was used as the pre-model for later DFT calculations, using OPLS-AA force field ${ }^{14)}$ as implemented in MacroModel software (Schrödinger Co.). The SGB model ${ }^{15,16)}$ was applied to consider the solvent effect. The first step of the study on ATP hydrolysis in HisP is to construct a model compound truncated from HisP structure to simplify the whole molecular system and make it treatable with DFT method, retaining the main characteristics of the system that may influence directly the ATP hydrolysis process.

To study the hydrolysis process, we performed an approximate saddle point search with the reaction coordinate as the distances between the $\gamma$ phosphorus atom $\left(\mathrm{P}_{\gamma}\right)$ of ATP and the oxygen atom of Wat437 $\left(\mathrm{O}_{\mathrm{W}}\right)$ in the model compound. The nucleophilic attack by Wat437 was studied by simulating the hydrolysis process of ATP that was triggered by pushing Wat437 toward $\mathrm{P}_{\gamma}$. Two more reaction coordinates were used to define the transition state (TS), one is the angle formed by $\mathrm{O}_{\mathrm{W}}, \mathrm{P}_{\gamma}$ and $\mathrm{O}$ atom of leaving group $\left(\mathrm{O}_{\beta \gamma}\right)$, denoted as angle $\mathrm{O}_{\mathrm{W}}-\mathrm{P}_{\gamma}-\mathrm{O}_{\beta \gamma}$ in the following; another one is dihedral angle among the planes formed by atoms $\mathrm{O}_{\gamma 1}$, $\mathrm{O}_{\gamma 2}, \mathrm{O}_{\gamma 3}$ and $\mathrm{P}_{\gamma}, \mathrm{O}_{\gamma 2}, \mathrm{O}_{\gamma 3}$ of ATP molecule, denoted as dihedral angle $\mathrm{O}_{\gamma 1}-\mathrm{O}_{\gamma_{2}}-\mathrm{O}_{\gamma 3}-\mathrm{P}_{\gamma}$, which are explained in the results and discussion.

\subsection{Model compound}

To study the hydrolysis of ATP in the binding site of HisP protein, the ATP binding site was truncated from energetic minimized crystallized structure, including ATP molecule, conserved residues (Gln100, Asp178, Glu179), interacting with triphosphate group of ATP molecule, water molecules surrounding triphosphate group are also kept as part of model components, one of which (Wat437) play a role of nucleophile during the hydrolysis mechanism, proposed by Okimoto et al. and Dittrich et al. on ATP hydrolysis in Myosin $^{17)}$ and F1-ATPase. ${ }^{18)}$ In this work, we focused on $\beta$ - and $\gamma$-phosphates similar to their works. Concretely the chemical groups extracted from the ATP bound protein to build the model compound were the tri-phosphate groups of ATP, the water molecules surrounding $\beta$ and $\gamma$ phosphates (Wat437, Wat415, and Wat500), the Glu179 $\mathrm{CH}_{3} \mathrm{COO}^{-}$ group, the Asp178 $\mathrm{CH}_{3} \mathrm{CH}\left(\mathrm{NH}_{3}\right) \mathrm{CO}^{-}$group, and the $\mathrm{Gln} 100$ $\mathrm{CH}_{3} \mathrm{COO}^{-}$group.

\subsection{Density functional theory calculations}

The geometry optimization and vibration frequency calculations for the model compound were performed within DFT scheme using the $\mathrm{DMol}^{3}$ program available from Material Studio (MS) package "version" 2.2 of Accelrys. ${ }^{19)}$ In each steps of the approximate saddle point search, distance constrained structures were optimized using the generalized gradient corrected functionals by Perdew and Wang (PW91) ${ }^{20)}$ the DND basis set (the double numerical basis sets augmented by a set of $d$ polarization functions), and the fine-quality mesh size for numerical integration. MS was also used to build and visualize the models and to visualize the eigenvector projections of the calculated vibrational modes of the models.

\section{Results and Discussion}

\subsection{Construction of the model compound}

Before the extraction of the components of the model compound, HisP protein was protonated and minimized. The minimization was performed in three steps: i) For hydrogen atoms, ii) for side chains and iii) for the entire protein.

The model compound was built by truncating pieces of structures from the minimized ATP-HisP system. Because divalent cations like $\mathrm{Mg}^{2+}$ and $\mathrm{Ca}^{2+}$ are necessary for ATP hydrolysis both in solution and when mediated by enzymes, ${ }^{21-23)}$ in the studied system, Wat407 was substituted by 


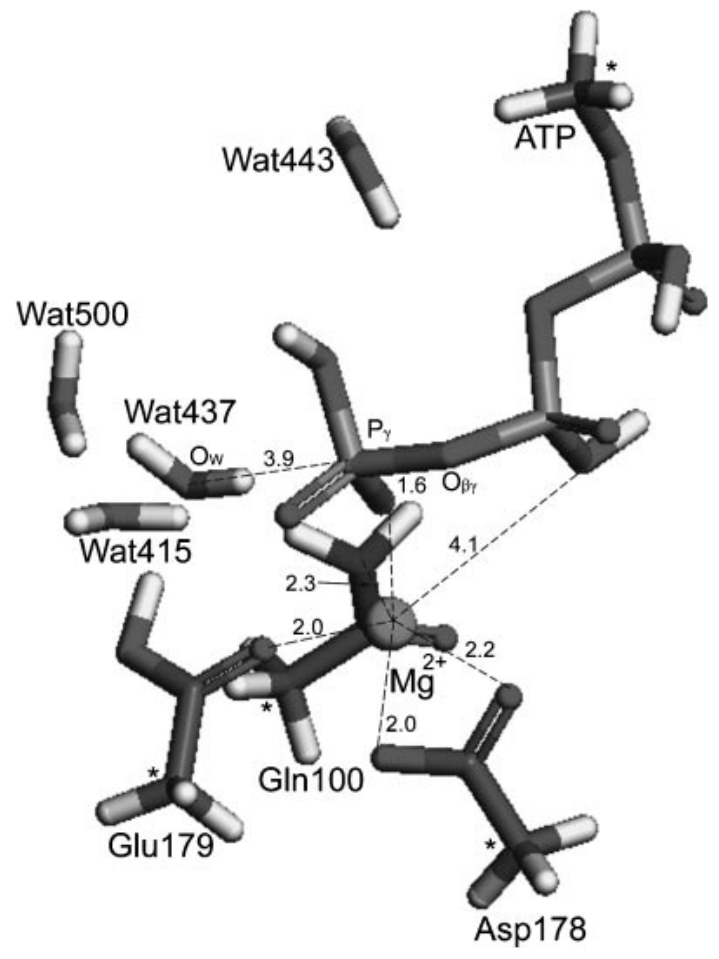

Fig. 2 Optimized model compound, which is used as initial structure for DFT calculations. The unit of distances shown in figure is $\AA$. The asterisked atoms are the ones, which are constrained during calculations to keep the shape of the binding site.

$\mathrm{Mg}^{2+}$ ion as recommended by Huang et al. ${ }^{13)}$ This position was found to be usually occupied by a divalent cation required for ATP hydrolysis. Since substitution of the Wat407 for $\mathrm{Mg}^{2+}$ may drive conformational changes in the structure, this was further optimized using DFT method. The optimized model compound including the respective $\mathrm{Mg}^{2+}$ cation is shown in Fig. 2, which is treated as initial structure for the following calculations. From the analysis of the substituted structure in comparison with non-substitution one, it was revealed that the phosphate groups of the ATP molecule together with Gln100, Asp178, and Glu179 of HisP protein interact electrostatically with $\mathrm{Mg}^{2+}$ cation forming a weak coordination complex, this is apparent from the distances of the $\mathrm{Mg}^{2+}$ to the $\mathrm{O}$ atoms of $\gamma$ phosphate group shown in Table 1.

As we mentioned in the method section, three reaction coordinates are used to define the TS of ATP hydrolysis. In the initial sate, the values of the three coordinates, which are distance of $\mathrm{O}_{\mathrm{W}}-\mathrm{P}_{\gamma}$, angle $\mathrm{O}_{\mathrm{W}}-\mathrm{P}_{\gamma}-\mathrm{O}_{\beta \gamma}$ and dihedral angle $\mathrm{O}_{\gamma 1}$ $\mathrm{O}_{\gamma 2}-\mathrm{O}_{\gamma 3}-\mathrm{P}_{\gamma}$, are $3.9 \AA, 162.3^{\circ}$ and $32.8^{\circ}$, respectively.

\subsection{ATP hydrolysis}

Several computational studies addressing the mechanism of ATP or guanosine tri-phosphate (GTP) hydrolysis in proteins have been carried out for systems other than NBDs of $\mathrm{ABC}$ transporter, as for $\mathrm{F}_{1}$-ATPase, myosin, $\mathrm{P} 21^{\text {ras }}$, and Cdc42. ${ }^{17,18,25-28)}$ An SN2-like nucleophilic attack mechanism was proposed in these cases. In order to demonstrate this hypothesis in the ATP-HisP protein system, we determined the conformations of the TS and product, which were obtained through an approximate saddle point search on TS
Table 1 Distances and angles in the initial, transition and product states.

\begin{tabular}{lccc}
\hline \multicolumn{4}{c}{ Record distances $(\AA)$ and angles $\left(^{\circ}\right)$} \\
\hline \multicolumn{1}{c}{ Distances $(\AA)$} & Initial state & Transition state & Product state \\
\hline $\mathrm{Mg}^{2+}-\mathrm{N}(\mathrm{Gln} 100)$ & 2.3 & 2.3 & 2.3 \\
$\mathrm{Mg}^{2+}-\mathrm{O} 1$ (Asp178) & 2.0 & 2.4 & 2.2 \\
$\mathrm{Mg}^{2+}-\mathrm{O} 2$ (Asp178) & 2.2 & 2.1 & 2.1 \\
$\mathrm{Mg}^{2+}-\mathrm{O}(\mathrm{Glu} 179)$ & 2.0 & 2.1 & 2.1 \\
$\mathrm{Mg}^{2+}-\mathrm{O}_{\gamma}$ & 2.0 & 2.2 & 2.3 \\
$\mathrm{Mg}^{2+}-\mathrm{O}_{\beta}$ & 4.1 & 2.2 & 2.2 \\
\hline $\mathrm{O}_{\mathrm{W}}-\mathrm{P}_{\gamma}$ & 3.9 & 1.8 & 1.6 \\
$\mathrm{P}_{\gamma}-\mathrm{O}_{\beta \gamma}$ & 1.6 & 2.0 & 3.5 \\
\hline $\left.\mathrm{Angles}^{\circ}{ }^{\circ}\right)$ & Initial state & Transition state & Product state \\
\hline $\mathrm{O}_{\mathrm{W}}-\mathrm{P}_{\gamma}-\mathrm{O}_{\beta \gamma}$ & 162.3 & 173.5 & 98.9 \\
Dihedral angle & 32.8 & -0.4 & -36.8 \\
\hline
\end{tabular}

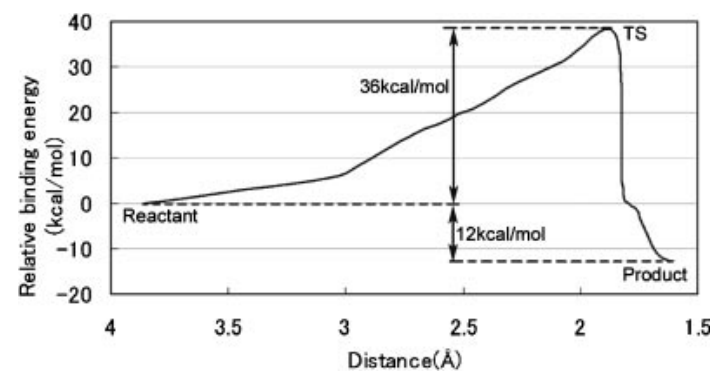

Fig. 3 Binding energies profile according to the approximate saddle point search along the reaction coordinate of $\mathrm{P}_{\gamma}-\mathrm{O}_{\mathrm{W}}$ distance.

by moving Wat437 towards the $\mathrm{P}_{\gamma}$ atom of ATP, i.e., decreasing the distance between $\mathrm{P}_{\gamma}$ and $\mathrm{O}_{\mathrm{W}}$ with a step of $0.2 \AA$. At each step we fully relaxed the system using DFT method constraining the $\mathrm{P}_{\gamma}-\mathrm{O}_{\mathrm{W}}$ bond and the positions of asterisked atoms in Fig. 2 to keep the shape of the binding pocket. The energies involved in the hydrolysis reaction were determined based on the calculations described above. Figure 3 shows the profile of relative binding energy against the reaction coordinate that is the distance between $\mathrm{O}_{\mathrm{W}}-\mathrm{P}_{\gamma}$, during the approximate TS search. The hydrolysis reaction energy, which was calculated by subtracting the binding energy of reactant from the one of product, was $-12 \mathrm{kcal} /$ $\mathrm{mol}$; and the reaction energy barrier, which was calculated by subtracting the binding energy of reactant from $\mathrm{TS}$, was $36 \mathrm{kcal} / \mathrm{mol}$.

\subsection{Transition state conformation}

Figure 4(a) shows the TS structure determined from our approximate saddle-point search based on DFT calculations. The TS structure shows the formation of $\mathrm{O}_{\mathrm{W}}-\mathrm{P}_{\gamma}$ partial bond with the bond length of $1.8 \AA$. Wat 437 acts as the attacking group, while ADP is acting as the leaving group. The bond length of $\mathrm{P}_{\gamma}-\mathrm{O}_{\beta \gamma}$ increased from $1.6 \AA$ in initial state to $2.0 \AA$ in TS state. On the other hand, TS structure also shows the interaction between the $\beta$ phosphate group of ATP molecule with the $\mathrm{Mg}^{2+}$ cation, in addition to the interactions in the initial state (Gln100, Asp178, Glu179 and $\gamma$ phosphate), forming a further coordinated complex that includes $\beta$ phosphate group of ATP, with weak ionic bonds to $\mathrm{Mg}^{2+}$. 


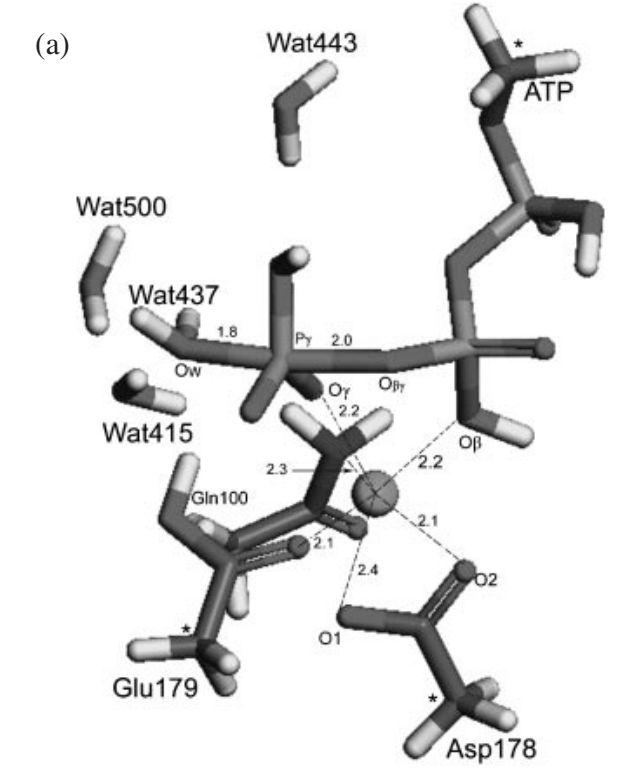

(b)

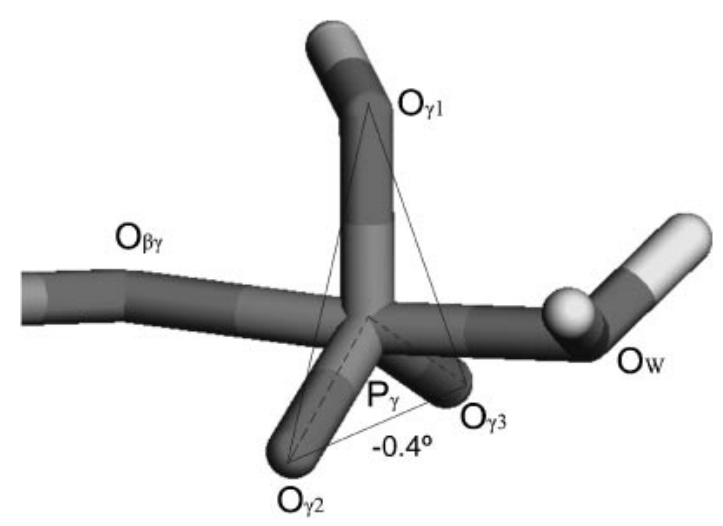

Fig. 4 (a) Transition sate structure of model compound, the distance between $\mathrm{P}_{\gamma}-\mathrm{O}_{\beta \gamma}$ and $\mathrm{P}_{\gamma}-\mathrm{O}_{\mathrm{W}}$ are 2.0 and $1.8 \AA$, respectively; (b) Two more reaction coordinates indicate a $\mathrm{SN} 2$ like reaction mechanism. One is the dihedral angle $\mathrm{O}_{\gamma 1}-\mathrm{O}_{\gamma 2}-\mathrm{O}_{\gamma 3}-\mathrm{P}_{\gamma}$, which is $-0.4^{\circ}$ in TS; and the other one is angle $\mathrm{O}_{\beta \gamma}-\mathrm{P}_{\gamma}-\mathrm{O}_{\mathrm{W}}$, which is $173.5^{\circ}$ in the TS.

Table 1 shows distances between $\mathrm{Mg}^{2+}$ and $\mathrm{O}_{\gamma}, \mathrm{O}_{\beta}$ of ATP, $\mathrm{N}$ atom of $\mathrm{Gln} 100, \mathrm{O}_{1}, \mathrm{O}_{2}$ of Asp178 and $\mathrm{O}$ of Glu179 in initial, transition and product states. The coordinated complex was stable in three states, indicating that even after ATP hydrolysis, products (ADP and phosphate group) are still kept in the binding site, which agrees with the proposed translocation mechanism of ABC transporter by Higgins. ${ }^{2)}$

In this study, two more reaction coordinates was introduced to define the same TS structure, as shown in Fig. 4(b), one is the dihedral angle $\mathrm{O}_{\gamma 1}-\mathrm{O}_{\gamma_{2}}-\mathrm{O}_{\gamma_{3}}-\mathrm{P}_{\gamma}$, which is $-0.4^{\circ}$ in $\mathrm{TS}$, and therefore almost planar; the other one is the angle $\mathrm{O}_{\mathrm{W}}-\mathrm{P}_{\gamma}-\mathrm{O}_{\beta \gamma}$, which reached its maximum value of $173.5^{\circ}$ in the same TS. Because that the planar TS is the typical feather of $\mathrm{SN} 2$ reaction, we proposed the interaction between $\mathrm{P}_{\gamma}$ atom and the attacking water molecule Wat437 exhibit a SN2-like mechanism.

\subsection{Product state conformation}

Moving Wat437 towards $\mathrm{P}_{\gamma}$ with the constrained optimization leads to the formation of the product, in which the

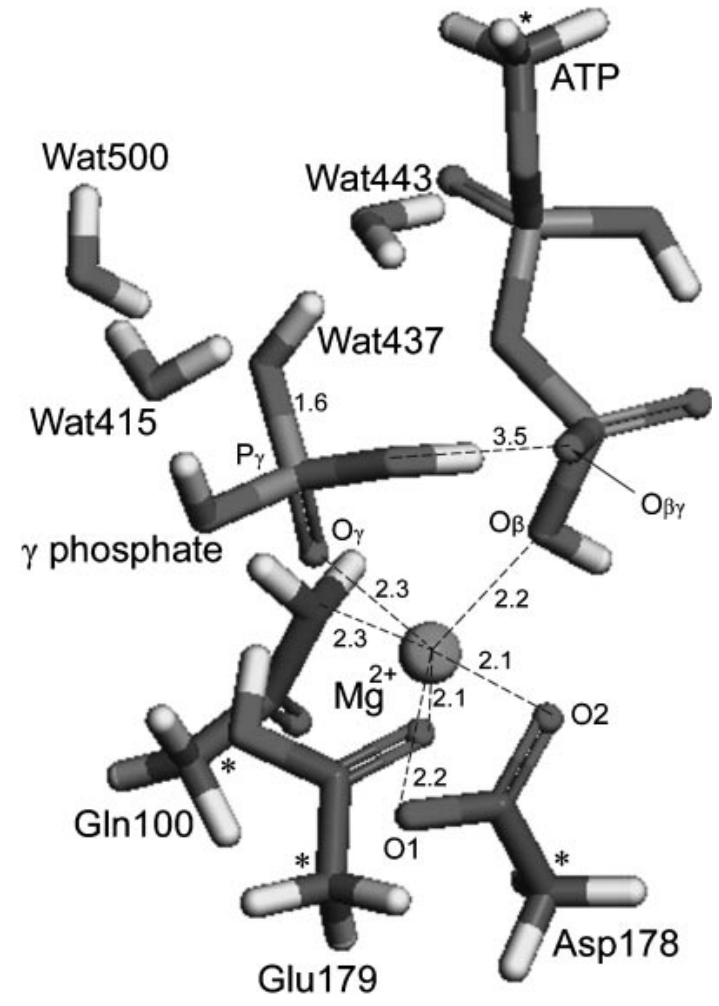

Fig. 5 Product state structure of model compound, ADP and Pi are products of ATP hydrolysis, which are kept inside the binding site after hydrolysis by forming the coordination complex with residues Gln100, Asp178 and Glu179 mediated by $\mathrm{Mg}^{2+}$.

ADP and $\gamma$ phosphate group moieties are even further separated. The angle $\mathrm{O}_{\mathrm{W}}-\mathrm{P}_{\gamma}-\mathrm{O}_{\beta \gamma}$ decreased step by step from $162.3^{\circ}$ in the initial state, maximum value of $173.5^{\circ}$ in TS until $98.9^{\circ}$ in the product state. One can observe in Fig. 5 that the covalent bond between $\mathrm{P}_{\gamma}$ and $\mathrm{O}_{\beta \gamma}$ is completely broken. This is deduced from the bond length that stretches from 1.7 to $3.5 \AA$. The phosphate group takes a tetrahedral conformation. ADP and the phosphate group do not, however, separate further due to the tight confinement by $\mathrm{Mg}^{2+}$, which is fixed by residues of the binding pocket, particularly by the side chains of Gln100, Asp178 and Glu179. Water molecules also contribute to forming the constrained conformation.

\subsection{Orbital interaction and electron transfer}

From orbital analysis, highest occupied molecular orbital (HOMO) of Wat437 was distributed by lone electron pair of water $\mathrm{O}$ atom. The lowest unoccupied molecular orbital (LUMO) of ATP molecule has a distribution on $\mathrm{P}_{\gamma}$, as shown in Fig. 6. The direction of this distribution is towards Wat437 (nuleophile), which supplied an opportunity to be attacked by HOMO of Wat437. We obtained an interacting molecular orbital between $\mathrm{O}_{\mathrm{W}}$ and $\mathrm{P}_{\gamma}$ in TS, the energy level of which is $-9.43 \mathrm{eV}$, lower than the energy level of Wat437 HOMO $(-6.95 \mathrm{eV})$. These evidences indicated a stabilized orbital interaction between Wat437 (nucelphile) and $\mathrm{P}_{\gamma}$ (electronphile). Mulliken charge analysis was performed on atoms involved in the reaction core of three states of initial, transition and product states, as shown in Table 2. The charges of $\mathrm{O}_{\beta \gamma}$ are $-0.71,-0.73$ and -0.72 in three state, respectively, while the charges of $\mathrm{O}_{\mathrm{W}}$ and $\mathrm{P}_{\gamma}$ are -0.59 , 


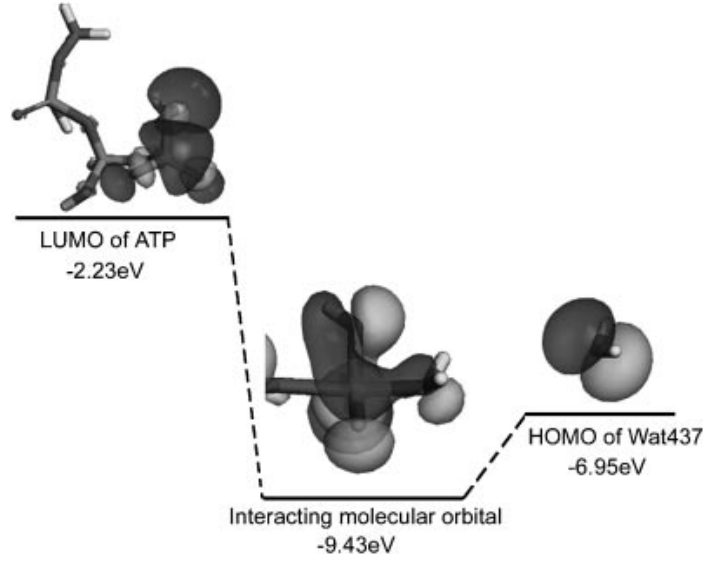

Fig. 6 Orbital interaction between HOMO of Wat437 and LUMO of ATP, the unit of energy level is au.

Table 2 Mulliken charge distribution analysis on Atoms of reaction core in initial, TS and product states.

\begin{tabular}{cccc}
\hline & Initial state & Transition state & Product state \\
\hline $\mathrm{P}_{\gamma}$ & 1.30 & 1.39 & 1.35 \\
$\mathrm{O}_{\mathrm{W}}$ & -0.59 & -0.46 & -0.59 \\
$\mathrm{O}_{\beta \gamma}$ & -0.71 & -0.73 & -0.72 \\
$\mathrm{O}_{\gamma 1}$ & -0.59 & -0.63 & -0.69 \\
$\mathrm{O}_{\gamma 2}$ & -0.72 & -0.71 & -0.67 \\
$\mathrm{O}_{\gamma 3}$ & -0.69 & -0.71 & -0.57 \\
\hline
\end{tabular}

$-0.46,-0.59,1.30,1.40$ and 1.35 , respectively. Based on the orbital and charge distribution analysis, we proposed that when the ATP hydrolysis occurs, electron transfers from $\mathrm{O}_{\mathrm{W}}$ (nucelphile) through $\mathrm{P}_{\gamma}$ (electronphile) to $\mathrm{O}_{\beta \gamma}$ (leaving group).

\section{Conclusions}

In the present work we have performed a detailed analysis of the hydrolysis reaction of ATP catalyzed by HisP protein. To study the reaction mechanism, a model compound was constructed from energy minimized HisP protein structure to perform DFT calculations. The calculations led us to propose an SN2-like nucleophilic attack mechanism for the hydrolysis of ATP catalyzed by HisP protein. Water molecules play a crucial role in SN2 mechanisms, particularly in this reaction, where a network of proton transfer proceeds through water molecules to $\gamma$ phosphate group has been proposed based on the calculations we have performed. Wat437 plays the role of nucleophile. During ATP hydrolysis, proton transfer through water molecules around the $\gamma$ phosphate group lead to the formation of the TS and the final product.

Our hypothesis is supported by several results from our calculations and agrees with other studies on similar systems. One such calculated value is the angle $\mathrm{O}_{\mathrm{W}}-\mathrm{P}_{\gamma}-\mathrm{O}_{\beta \gamma}$, which is formed by the attacking and leaving oxygen with $\gamma$ phosphors atom, decreased from $162.3^{\circ}$ in the initial state, $173.5^{\circ}$ in TS until $98.9^{\circ}$ in product state continuously, while $\mathrm{P}_{\gamma}-\mathrm{O}_{\beta \gamma}$ distance increased from $1.6 \AA$ in initial state to $3.5 \AA$ in the product state, this unambiguously indicates that $\mathrm{P}_{\gamma}-\mathrm{O}_{\beta \gamma}$ bond is broken and it is associated with the rotation of the $\gamma$ phosphate group. The binding of $\mathrm{Mg}^{2+}$ to the $\gamma$ phosphate groups and the protein environment hamper the rotation of the hydrolyzed $\gamma$ phosphate group, which stays in the binding site after hydrolysis. This is confirmed with the hypothesis of Higgins et al. (2004), ${ }^{2)}$ who assumed that phosphate group remains in the binding groove of NBDs after ATP hydrolysis.

\section{REFERENCES}

1) C. A. Doige and G. F. Ames: Annu. Rev. Microbiol. 47 (1993) 291319.

2) C. F. Higgins and K. J. Linton: Nat. Struct. Mol. Biol. 11 (2004) 918926.

3) I. Carrier and M. Julien: Biochemistry 42 (2003) 12875-12885.

4) G. F. Ames and E. N. Spudich: Proc. Natl. Acad. Sci. U.S.A. 73 (1976) 1877-1881.

5) R. E. Kerppola and V. K. Shyamala: J. Biol. Chem. 266 (1991) 98579865.

6) K. Nikaido and P. Q. Liu: J. Biol. Chem. 272 (1997) 27745-27752.

7) C. E. Liu and G. F. Ames: J. Biol. Chem. 272 (1997) 859-866.

8) K. Nikaido and G. F. Ames: J. Biol. Chem. 274 (1999) 26727-26735.

9) D. I. Kreimer and H. Malak: Eur. J. Biochem. 267 (2000) 4242-4252.

10) G. F. Ames and K. Nikaido: J. Bioenerg. Biomembr. 33 (2001) 79-92.

11) P. M. Jones and A. M. George: Proc. Natl. Acad. Sci. U.S.A. 99 (2002) 12639-12644.

12) J. D. Campbell and S. S. Deol: Biophys. J. 87 (2004) 3703-3715.

13) L. W. Huang and I. X. Wang: Nature 396 (1998) 703-707.

14) W. L. Jorgensen and D. S. Maxwell: J. Am. Chem. Soc. 118 (1996) 11225-11236.

15) L. Zhang and E. Gallicchio: J. Comp. Chem. 22 (2001) 591-607.

16) A. Ghosh and C. S. Rapp: J. Phys. Chem. B 102 (1998) 10983-10990.

17) N. Okimoto and K. Yamanaka: Biophys. J. 81 (2001) 2786-2794.

18) M. Dittrich and S. Hayashi: Biophys. J. 85 (2003) 2253-2266.

19) B. Delley: J. Chem. Phys. 113 (2000) 7756-7764.

20) Y. Wang and J. P. Perdew: Phys. Rev. B 44 (1991) 13298-13307.

21) A. V. Syroeshkin and M. A. Galkin: Biochemistry Mosc. 64 (1999) 1128-1137.

22) W. Chen and G. Guidotti: BMC Biochem. 2 (2001) 9.

23) E. O. Oloo and D. P. Tieleman: J. Biol. Chem. 279 (2004) 4501345019.

24) C. Orelle and O. Dalmas: J. Biol. Chem. 278 (2003) 47002-47008.

25) T. M. Glennon and J. Villá: Biochemistry 39 (2000) 9641-9651.

26) R. Langen and T. Schweins: Biochemistry 32 (1992) 8691-8696.

27) T. Schweins and R. Langen: Nat. Struct. Biol. 1 (1994) 476-484.

28) A. Cavalli and P. Carloni: J. Am. Chem. Soc. 124 (2001) 3763-3768. 